\title{
Zur Kenntniss der Milzsyphilis.
}

\author{
Von Dr. L. Gold aus Odessa.
}

Aus Dr. Chiari's Prosectur im Rudolfspitale in Wien.

(Hierzu TafeI X.)

Während die durch die Syphilis gesetzten Veränderungen in vielen Organen des menschlichen Körpers, so in der Haut, in den angrenzenden Schleimhautpartien, in den Knochen und in anderen in anatomischer Hinsicht gewöhnlich so charakteristisch sind, dass ihre Diagnose meist ganz sicher gemacht werden kann, gibt es Organe, in denen die bestimmt als syphilitiseh zu bezeichnenden Erkrankungen sebr selten sind. $\mathrm{Za}$ diesen Organen gehört auch die Milz. Man findet sie zwar oft genug in den Leichen syphilitisch gewesener Individuen verändert, jedoch sind diese Veränderungen meist so beschaffen, dass sie auch aus anderen Ursachen, als aus der Syphilis ihren Ursprung nehmen kounten. Die in solchen Fällen constatirte chronische Intumescenz des Organs, Verdickung der Trabecularsubstanz, Schwielenbildung in der Kapsel, narbige Einziehung an der Oberfläche und amyloide Entartung des Parenchyms mögen wohl häufig mit der Syphilis im Zusammenhang stehen, jedoch ist es gewiss sehr schwer, sie nach dem gegenwärtigen Stande unserer anatomischen Kenntnisse von der Syphilis als sicher syphilitischen Ursprunges aufzufassen. Anf derartige Veränderungen beziehen sich auch die meisten Publicationen, welche man in der anatomischen Literatur der Milzsyphilis findet. Eine ganze Reihe von Autoren, wie Lecorché ${ }^{1}$ ), Pleischl

') Schmidt's Jahrbücher, 185\%, Bd. 94, p. 64 . 
und Klob ${ }^{1}$ ), Meyer ${ }^{2}$ ), Eberth ${ }^{3}$ ), Berkeley Hill ${ }^{4}$ ), S. Gee ${ }^{5}$ ), Wetzlar ${ }^{6}$ ), Hedenius ${ }^{5}$, Barlow ${ }^{8}$ ), Mosler ${ }^{9}$ ) und Ludwig Eürth ${ }^{10}$ ) haben an der Milz constitutionell Syphilitischer Intumescenz, Schwielenbildung und Verdickung der Kapsel gefunden. Andere Autoren, wie Dittrich ${ }^{11}$ ), Hecker ${ }^{12}$ ), Friedreich ${ }^{13}$ ), Hilton ${ }^{14}$ ), Alrik Ljunggren ${ }^{15}$ ), Oser ${ }^{16}$ ) and Löwenfeld ${ }^{17}$ ) trafen die Milz bei hereditärer und acquirirter constitutioneller Syphilis speckig und amyloid entartet.

In der gleichen Weise habe auch ich Gelegenheit gehabt, an der Haud der Sectionsprotokolle des Rudolfspitales vom December 1879 bis jetzt über die Häufigkeit zwar pathologischer, aber nicht bestimmt als syphilitisch sich darstellender Milzbefunde bei in dieser Zeit secirten Leichen constatirt syphilitisch Gewesener Erfahrung zu sammeln. Nachstehende Tabelle gibt das Datum der Section, das Alter und Geschlecht der Individuen, den pathologischen Befund überhaupt und den speciellen Milzbefund ron 10 solchen Fällen an.

1) Wiener medicinische Wochenschrift, 1860, Nr. 8, 9 und 10.

2) Schmidt's Jahrbücher, 1862, Bd. 114, p. 312.

3) Virchow's Archiv, 1867, Bd. XL, p. 326.

) Syphilis and local contagious disorders, London 1868, p. 130.

5) Vielfach citirt.

5) Canstatt's Jabresberichte, 1869, Bd. II, p. 558 .

7) Vierteljahrsschrift für Dermatologie und Syphilis, 1876, p. 647.

3) Dentsche raedicinische Wochenschrift, 1877, p. 201.

7) Ziemssen's Handbueh der speciellen Pathologie und Therapie, 8. Bd., 2. Hälfte, p. 142 .

10) Die Pathologie und Therapie der hereditären Syphilis étc. 1879 , p. 58 und 77 .

11) Prager Viertelj, 1849, Bd. I, p. 20.

12) Schmidt's Jahrbucher, 1856, Bd. 89, p. 309.

$\left.{ }^{13}\right)$ Virchow's Archiv, 1857, Bd. XI, p. 387 .

${ }^{14}$ S Schmidt's Jahrbücher, 1866, Bd. 130, p. 295.

$\left.{ }^{15}\right)$ Archiv für Dermat. und Syphilis, 1870, p. 547.

$\left.{ }^{10}\right)$ Arehiv für Dermat. und Syphilis, 1871, p. $2 \%$.

si) Canstatt's Jahresb. 1873, Bd. II, p. 58\%. 


\begin{tabular}{|c|c|c|c|}
\hline $\begin{array}{l}\text { Datum } \\
\text { der } \\
\text { Section }\end{array}$ & $\begin{array}{c}\text { Alter } \\
\text { und Ge- } \\
\text { schlecht }\end{array}$ & Pathologischer Befund iberhaupt & Milzbefund \\
\hline $\begin{array}{c}\text { 17. De- } \\
\text { cember } \\
1879\end{array}$ & $\begin{array}{c}56 \text { Jahre } \\
\text { alte } \\
\text { Frau }\end{array}$ & $\begin{array}{l}\text { Pneumonia crouposa. Pericarditis } \\
\text { incipiens. Ozaena syphilitica. } \\
\text { Periostitis syphilitica cranii et } \\
\text { tibiarum. Ulcera syphilitica } \\
\text { palati molli }\end{array}$ & $\begin{array}{l}\text { Die Milz etwas grösser, } \\
\text { ihre Kapsel stellenweise } \\
\text { zu knorpelartigen Höckern } \\
\text { umgewandelt. }\end{array}$ \\
\hline $\begin{array}{c}24 . \\
\text { Jämner } \\
1880\end{array}$ & $\begin{array}{l}\text { 56 Jahre } \\
\text { alte } \\
\text { Frau }\end{array}$ & $\begin{array}{l}\text { Tuberculosis obsoleta apicis pulm. } \\
\text { sin. Tuberculosis acuta pulm. } \\
\text { et peritonei. Cicatrices et ulce- } \\
\text { rationes syphiliticae cutis. Ulce- } \\
\text { ratio syph. laryngis et pharyng. }\end{array}$ & $\begin{array}{l}\text { Die Milz etwas grosser, } \\
\text { locker. }\end{array}$ \\
\hline $\begin{array}{c}24 . \\
\text { Jänner } \\
1880\end{array}$ & $\begin{array}{l}\text { 36 Jahre } \\
\text { alte } \\
\text { Fran }\end{array}$ & $\begin{array}{l}\text { Syphilis inveterata (pharyngis, la- } \\
\text { ryngis et nasi). Degeneratio } \\
\text { amyloidea. Hydrops universalis }\end{array}$ & $\begin{array}{l}\text { Die Milz um die Hälfte } \\
\text { grösser, derb, auf dem } \\
\text { Durchschnitt stark glän- } \\
\text { zend. - Mikroskop.: ihr } \\
\text { Parenehym beträchtlich } \\
\text { amyloid. }\end{array}$ \\
\hline $\begin{array}{l}\text { 2. April } \\
1880\end{array}$ & $\begin{array}{l}\text { 31 Jahre } \\
\text { alter } \\
\text { Mann }\end{array}$ & $\begin{array}{l}\text { Carcinoma medullare ductus cho- } \\
\text { ledochi cum stenosi hujus et } \\
\text { dilatatione ductuou bilifero- } \\
\text { rum. Icterus universalis. Car- } \\
\text { cinoma secund. hepatis. Hae- } \\
\text { morrhagiae textus cellulosi. } \\
\text { Syphilis (cicatrix penis, pig- } \\
\text { mentationes cutis). }\end{array}$ & $\begin{array}{l}\text { Die Milz am die Hälfte } \\
\text { grösser, derb. }\end{array}$ \\
\hline $\begin{array}{l}25 . \\
\text { April } \\
1880\end{array}$ & $\begin{array}{l}\text { 33 Jahre } \\
\text { alter } \\
\text { Mann }\end{array}$ & $\begin{array}{l}\text { Tuberculosis chronica apicum pul- } \\
\text { monum et intestini erassi. Tu- } \\
\text { berculosis subacuta pulmonum. } \\
\text { Dysenteria. Arthritis deformans } \\
\text { articulationis cubiti dextrae. } \\
\text { Syphilis (cicatrices, pigmenta- } \\
\text { tiones et ulcera cutis, ulcera } \\
\text { pharyngis). }\end{array}$ & $\begin{array}{l}\text { Die Milz etwas dichter, aber } \\
\text { nicht vergrössert. }\end{array}$ \\
\hline $\begin{array}{l}\text { 7. Mai } \\
1880\end{array}$ & $\begin{array}{l}\text { 51 Jahre } \\
\text { alter } \\
\text { Mann }\end{array}$ & $\begin{array}{l}\text { Dysenteria follicularis. Phthisis } \\
\text { tuberculosa pulmonum et inte- } \\
\text { stini. Morbus Brightii chronic. } \\
\text { Concretio cordis cum pericardio. } \\
\text { Syphilis (hyperostosis cranii, } \\
\text { cicatrices hepatis, glandis penis } \\
\text { et reg. inguin. dextrae) }\end{array}$ & $\begin{array}{l}\text { Die Milz aufs doppelte ver- } \\
\text { grőssert, Kapsel rerdickt, } \\
\text { ihr Parenchym blutreich. }\end{array}$ \\
\hline $\begin{array}{c}8 . \mathrm{Mai} \\
1880\end{array}$ & $\begin{array}{l}\text { So Jahre } \\
\text { alter } \\
\text { Mann }\end{array}$ & $\begin{array}{l}\text { Tuberculosis chronica pulmonum, } \\
\text { tracheae, laryngis et genitalis. } \\
\text { Cicatrices syphiliticae pharyn- } \\
\text { gis. Oedema glottidis }\end{array}$ & $\begin{array}{l}\text { Die Milz blass, nicht wei- } \\
\text { ter verändert. }\end{array}$ \\
\hline $\begin{array}{c}\text { 9. Mai } \\
1880\end{array}$ & $\begin{array}{l}\text { 30 Jahre } \\
\text { alte } \\
\text { Frau }\end{array}$ & $\begin{array}{l}\text { Erysipelas faciei. Syphilis (exan- } \\
\text { thema maculo-papulosum cutis, } \\
\text { condylomata lata ad genitalia, } \\
\text { intumescentia glandulavum } \\
\text { lymph. univer alis). }\end{array}$ & $\begin{array}{l}\text { Die Milz ar ein geringes } \\
\text { vergrossert, blass, locker. }\end{array}$ \\
\hline
\end{tabular}




\begin{tabular}{|c|c|c|c|}
\hline $\begin{array}{l}\text { Datum } \\
\text { der } \\
\text { Section }\end{array}$ & $\begin{array}{c}\text { Alter } \\
\text { und Ge- } \\
\text { schlecht }\end{array}$ & Pathologischer Befund überhaupt & Milzbefund \\
\hline $\begin{array}{l}\text { 17. } \\
\text { Juni } \\
1880\end{array}$ & $\begin{array}{c}36 \text { Jahre } \\
\text { alte } \\
\text { Frau }\end{array}$ & $\begin{array}{l}\text { Tubereulosis chronica pulmonum. } \\
\text { Pleuritis tubercul. haemorrlag. } \\
\text { sinistra. Syphilis (exanthena } \\
\text { papulosum cutis, hypernstosis } \\
\text { tibiarum, intumescentia glan- } \\
\text { dularum lymphaticartm, stric- } \\
\text { tura recti). } \\
\end{array}$ & $\begin{array}{l}\text { Die Milz sehr innig an der } \\
\text { Nachbarschaft adhärent; } \\
\text { Kapsel verdickt, besonders } \\
\text { an der convexen Fläche } \\
\text { des oberen Poles, woselbst } \\
\text { sich eine etwa } 6 \square \text { Cent. } \\
\text { grosse bis } 3 \text { Millim. dicke } \\
\text { schwielige Platte in der } \\
\text { Kapsel vorfindet. Die Milz } \\
\text { bedeutend grösser, } 18 \text { Ctm. } \\
\text { lang, 10 Ctm. breit, bis } 3^{3} / 2 \\
\text { Ctm. dick, 30oGm, schwer. } \\
\text { Ihr Parenchym dicht, mäs- } \\
\text { sig mit Blut versehen. }\end{array}$ \\
\hline $\begin{array}{l}17 \\
\text { Jini } \\
1880\end{array}$ & $\begin{array}{l}27 \text { Jahre } \\
\text { alte } \\
\text { Fran }\end{array}$ & $\begin{array}{l}\text { Phthisis tuberculosis prlmonum. } \\
\text { Strictura recti (syphilitiea), Pe- } \\
\text { riproctitis. }\end{array}$ & $\begin{array}{l}\text { Die Milz etwas vergrös- } \\
\text { sert, blass. }\end{array}$ \\
\hline
\end{tabular}

Wenu man in dieser Tabelle die Milzbefunde überblickt, so sieht man, dass zwar die Milz meistens (9 mal in den zehn Fällen) pathologisch verändert war, dass jedoch die Diagnose dieser Veränderungen als syphilitischer Erkrankungen gewiss nicht eine sichere genannt werden kann, indem $j a$ auch sonst oft bei den Obduetionen die Milz in diesen Formen pathologisch angetrofien wird.

Ganz im Gegensatze zu diesen häufig wahrzunehmenden pathologischen Milzveränderungen überhaupt in den Leichen syphilitiseh Gewesener ist der Befund der eigentlichen anatomisch wohl charakterisirten syphilitischen Gummen der Milz ein sehr seltener, so dass ich mir deswegen erlauben will, sowohl die mir bekannt gewordenen bezüglichen Mittheilungen aus der Literatur hier vorzubringen, als auch des Genaueren über einen derartigen Fall zu berichten, den Herr Prosector Docent Dr. H. Chiari am 11. Jänner dieses Jahres im Rudolfspitale secirte und mir zur Bearbeitung überliess.

Bereits Rokitansky erwähnt bei der Besprechung der Lebersyphilis ${ }^{1}$ ) das Vorkommen von syphilitischen Schwielen und

t) Lehrbuch der pathologischen Anatomie, 3. Auflage, 3. Bd., p. 254. 
verkästen Knoten in der Milz. Virchow ${ }^{1}$ ) fand in manchen Fällen in der Milz constitutionell-Syphilitischer gelbe, bärtere Stellen, die bei der mikroskopisehen Untersuchung wenig Pigment, dagegen viel Fett erkennen liessen und die sich daher seiner Ansicht nach mebr den gummösen, als den hämorrhagischen Knoten anreihten.

Biermer ${ }^{2}$ ) beschreibt zwei Fälle von Milzgummata bei constitutioneller Syphilis. Der erste Fall betraf eine 41jährige Fran mit constatirter Syphilis. Die Milz ersehien vergrössert, die gummösen Wucherungen waren linsengross und flach und lagen nahe an der Kapsel. Der zweite Fall betraf eine 46jährige Frau mit constitutioneller Syphilis und ulcerösem Syphilid. In der vergrösserten und gelappten Milz fanden sich 6 gummöse Knoten im Durchmesser von $0.5-1.0 \mathrm{Ctm}$., welche grösstentheils in regressiver Metamorphose begriffen waren. - Dufeillay ${ }^{3}$ ) fand in der Milz eines mit pustulösem and tuberculösem Syphilid behaftet gewesenen syphilitischen Mannes mehrere gummöse Knoten mit käsigem Zerfall im Centrum. Wagner ${ }^{4}$ ) sah Gummata in der Milz 9mal, von denen er 4 Fälle ausführlich mittheilt. In diesen 4 Fällen fand Wagner in der vergrösserten Milz zahlrejche Knoten von kaum Hirsekorngrösse bis 2-6" im Durchmesser, von grauweisser und grauröthlicher Farbe und unregelmässiger Gestalt. Die Knoten waren von dem übrigen Milzgewebe mehr weniger scharf, aber nicht kapselartig abgegrenzt und heller, derber und trockener, als das übrige Milzgewebe. Forster und Rees ${ }^{5}$ ) fanden in der Milz von Leichen Syphilitischer sehr grosse und mehrere zerstreute kleinere Knoten, welche sie als gummöse Knoten bezeichnen. Samuel Willks ${ }^{6}$ ) theilt einige unzweifelhafte Fälle mit, in welchen syphilitische Ablagerungen anch in anderen Organen vorhanden waren. Er fand in der Milz umschriebene Knoten nahe der Oberfläche der Kapsel. Die Knoten waren gelb und bestanden bei mikroskopischer Untersuchung aus Fasern, homogener Substanz

") Virchow's Archiv, 1858, Bd. XV, p. 319.

$\left.{ }^{2}\right)$ Schweizerische Zeitschrift für Heilkunde, 1862, I., p. 118.

3) Schmidt's Jahrbücher, 1863, Bd. 118, p. 41.

4) Archiv für Heilkunde, 1863, Bd. IV, p. 430 .

b) Sehmidt's Jahrbucher, 1863, Bd. 118, p. 40.

5) Schmidt's Jahrbücher, 186\%, Bu. 193, n. 17\%. 
und Fettkörnchen. - Arnold Beer ${ }^{1}$ ) stimmt mit anderen Forschern ganz überein, dass die Milzsyphilis eine grosse Variabilität besitze, dass sie entweder als diffuse Hyperplasie und zwar sehr oft oder viel seltener in Form von circumscripten Milzherden, nämlich Käseknoten vorkomme. Letztere Form beschreibt Beer in einigen Fällen constatirter Syphilis. - Gregoric ${ }^{2}$ ) fand in der durch narbige Einziehungen verkleinerten Milz eines 36jährigen syphilitischen Mannes mebrere Gummaknoten, die die verschiedensten Stadien der Entwicklung hatten. - Hutchinson und Jackson ${ }^{8}$ ) beobachteten zwei Fälle, in denen im Parenchym der Milz gummöse Knoten waren. - Nach Bäumler ${ }^{4}$ ) sah Zenker in der Milz syphilitisehe Gummaknoten. - Birch-Hirschfeld ${ }^{5}$ ) beschreibt in der syphilitischen Milz Schwielen, in denen sich käsige Knoten finden.

Nebst anderen bereits erwähnten Fällen citirt Jullien ${ }^{6}$ ) einen Fall von Alling, welchen derselbe im Jahre 1869 in einer anatomischen Gesellschaft vorstellte. In der Milz fanden sich mehrere Gummata von verschiedener Grösse.

Diesen soeben citirten Fällen gummöser Milzsyphilis will ich nun den früher erwähnten neuen Fall anfügen.

Derselbe bezieht sich auf eine 45jährige Frau, K. W., welche von der Abtheilung des Herrn Prof. Prim. Dr. v. Schrötter unter der Diagnose: „Tuberculosis pulmonum, Strictura recti“ zur Obduction gekommen war.

Seit 17 Jahren hatte dieselbe an Syphilis gelitten und war deswegen zu wiederholten Malen im Rudolfspitale behandelt worden. Die Syphilis hatte sich theils in Form von Hautsyphiliden und zwar auch ulceröser Natur, theils in Form einer zu Strictur führenden Verschwärung im Rectum manifestirt. und 160 .

1) Die Eingeweide-Syphilis. Tubingen 4867, p. 7, 91, 121, 127, 130

") Schmidt's Jahrbücher, 1871, Bd. 18̈1, p. 29. philis, 1876.

8) Citirt von Lancéreaux, Traité historique et pratique de la Sy-

") Ziemssen's Handbuch der speciellen Pathologie und Therapie, Bd. III, p. 184 .

5) Lehrbuch der pathologischen Anatomie, 1876, p. 4 \%月.

${ }^{6)}$ Traité pratique des maladies vénériennes. Paris 1879, p. 920. 
Die circa 30 Stunden post mortem vorgenommene Antopsie ergab folgenden Befund:

„Der Körper klein, schwächlich, abgemagert, blass, ictorisch, mit reichlichen Todtenflecken rückwärts. Das Haar grau; Pupillen enge; Thorax schmal; Unterleib wenig ausgedehnt.

Die weichen Schädeldecken blass; das Schädeldach compact, im Stirntheile etwas dicker. Die Meningen und das Gehirn blass.

In der Luftröhre serös-schleimige Flüssigkeit; ihre Schleimhaut wie die des Kehlkopfes und des Phargnx blass. Beide Lungen im ganzen Umfange angewachsen, im Allgemeinen gedunsen, leicht odematös, in den Spitzen durch alte Schwielen, in die käsige Knoten eingeschlossen sind, verdichtet. In der rechten Lungenspitze überdies eine wallnussgrosse Caverne.

Das Herz von gewöhnlicher Grösse. In der Bauchhöhle etwa $150 \mathrm{CCm}$. dicklichen, eitrigen Exsudates. - Das Peritonäum getrübt, mit fibrinös-eitrigen Exsudatlamellen bedeckt.

Die Leber blass, in ihrer Blase reichliche, dunkle Galle. Der Ductus cysticus und hepaticus zwar durchgängig, jedoch durch schleimig-eitrige Flüssigkeit verlegt.

In der Milz Gummata. (Der bezügliche ausführliche Befund wird später mitgetheilt werden.)

Die beiden Nieren bleich. In der Harnblase sehr wenig Harn.

Der Uterus in seinem Fundus und Corpus durch mehrere in die Wand eingelagerto, bis hühnereigrosse Myome missstaltet. Die Adnexa uteri mit der Nachbarschaft verwachsen. In der Vulva und Urethra alte Narben.

Der Magen ziemlich stark ausgedehnt; in demselben gallig gefärbter Schleim. Der Dünndarm zusammengezogen; in ihm wenig chymöse Stoffe. Im Dickdarme spärliche Scybala. Das Rectum in seiner $15 \mathrm{Ctm}$. langen unteren Partie der Schleimhaut durch Ulcera. tion vollständig verlastig, in seiner Wand schwielig verdichtet und so stenosirt, dass gerade nur der Zeigefinger durchgoführt werden kann. Das verdichtete periproctale Zellgewebe daselbst im hohen Qrade eitrig-janchig infiltrirt. In den höheren Antheilen des Rectums und im $\mathrm{S}$. Romanum etliche bis kreuzerstäckgrosse, rundliche, jetzt zum Theile in Heilung begriffene Substanzverluste.

An den Tibien keine Hyperostose zu finden. Auf dom Durehschnitte des rechten Femur und der rechten Tibia nichts Abnormes. 
Bei der mikroshopischen Untersuchung der Leber, Nieren und Milzknoten keine Amyloiddegeneration zu constatiren."

Diagnose: „Strictura recti (syphilitica) subsequente periproctitide et peritonitide purulenta diffusa. Gummata lienis. Tuberculosis obsoleta apicum pulmonum."

Die Untersuchung der Milz erwies das Organ als nicht vergrössert. Dasselbe mass $11 \mathrm{Ctm}$. in der Länge, $5.5 \mathrm{Ctm}$. in der Breite und $4 \mathrm{Ctm}$. in der Dicke. Seine Kapsel war glatt und im Allgemeinen nur unbedeutend verdickt. An der convexen Fläche des unteren Poles zeigte sie eine etwas stärkere Verdickung und war bier im Bereiche einer $4 \square$ Ctm. grossen Fläche in eine bis $2 \mathrm{Mm}$. dicke fibröse Platte umgewandelt. Bereits von aussen liess sich im oberen Ende der Milz nahe dem Hilus die Existenz eines über wallnussgrossen, harten, unregelmässig geformten Knotens constatiren. Das bestätigte auch der in der gewöhnlichen Weise von der convexen Fläche aus geführte mediane Längsschnitt, indem sich jetzt eine in das Milzparenchym eingebettete, bis an die Kapsel des Hilus heranreichende, nahezu ovoide Geschwulst zeigte, welche circa $5 \mathrm{Ctm}$. lang und $2 \cdot 5 \mathrm{Ctm}$. breit war (vide Fig. $1 \mathrm{a}$ ). Von der Oberfläche des oberen Milzpoles war sie nur wenigo $\mathrm{Mm}$. weit entfernt, ja erreichte sogar stellenweise daselbst die Kapsel, von der der Mitte ihrer Länge entsprechenden Partie der convexen Milzfäche war sie $2.5 \mathrm{Ctm}$. und von der Oberfläche des unteren Milzpoles $5.5 \mathrm{Ctm}$. weit entfernt. Thre Begrenzung gegen das übrige Milzgewebe war in ihrer unteren Hälfte eine zwar zackige, aber doch ziemlich scharfe, nach oben hin ging sie ganz allmälig in letzteres über. Die Geschwulst selbst bestand aus mehreren, kleineren, mit einander verschmolzenen und so eben nur theilweise mehr von einander zu sondernden Knoten, welche der Hauptsache nach aus Narbengewebe zusammengesetzt waren, jedoch allenthalben in das Narbengewebe eingestreut, theils scharf, theils undentlich abgegrenzte käsige Herde enthielten (vide Fig. $1 b$ ). An einzelnen Stellen fand sich im Narbengewebe auch gellbbraunes Pigment eingelagert, ferner zeigten sich in dem Durchschnitte durch die Geschwalst etliche grössere, nicht thrombosirte, sondern noch sondirbare Blutgefässstämme. Das übrige Milzparenchym war etwas derber als normal, dabei blass. Sehr interessant ge- 
stalteten sich die histologischen Verhältnisse der Knoten, die an Schnitten derselben nach Erhärtung in Alkobol und Färbung in Carmin studirt wurden. Figur 2 stellt einen solchen durch die Grenzzone zwischen den Knoten in der Milz und dem angrenzenden Milzparenchyme geführten Durchschnitt bei schwacher Vergrösserung (10malig) dar. Die dunkel schattirten Stellen bei $a$ sind die käsigen Herde, um sie herum die Schwiele und an letztere angrenzend bei $b$ das Milzparenchym. Die Sehwiele zeigte verschiedene Besebaffenheit, je nach ihrer Distanz von den käsigen Herden, indem sie in der unmittelbaren Nachbarschaft derselben sehr dicht und zellenarm, in weiterer Entfernung von den käsigen Herden, in der Nähe des Milzparenchyms hingegen als sebr zellenreich sich darstellte. Diese Differenz der Beschaffenheit der Sehwiele in den mehr centralen und peripheren Partien eines Knotens zeigt Fig 3 deutlich, indem bei $a$ die zellenarme, bei $b$ die zellenreiche Schicht wiedergegeben ist. Die Arterien und Venen hatten im Bereiche der Geschwulst in der Milz fast durchwegs eine auffallend starke Intima mit stellenweiser Wucherung des Endothels. An den Capillaren war jedoch nur zum geringsten Theile Endothelwucherung zu sehen. Bei genauerer Besichtigung der Grenzzone der schwieligen. Knoten gegen das übrige Milzparenchym liess sich. deutlich erkennen, dass die Schwiele aus den sogenannten Zellenbalken der Milz hervorgegangen war, indem nämlich dieselben, wie dies Fig. 4 erkennen lässt, durch allmälige Vermehrung der in ihnen enthaltenen Zellen in die periphersten Antheile des Gummas sich umwandelten. Die Venensinus verödeten dabei vollständig. Die Trabekel in den Knoten waren sehr wenig verdickt, wie das auch Wagner (l. c. pag. 434) für seine Fälle angibt, indem er sagt, dass das Milzsyphilom am wahrscheinlichsten vorzugsweise aus dem Milzgewebe und nur theilweise aus den fibrösen Balken und der Adventitia der Arterien entsteht.

Nach dem Mitgetheilten zeigt sich, dass die Geschwulst in der Milz dieses Falles durch eine Gruppe schwieliger Knoten, die an sich käsige Herde enthalten, gebildet wird. Das ist aber ganz derselbe Befund, wie bei den so oft vorkommenden älteren Gummaknoten der Leber, so dass also in vorliegendem Falle die Diagnose auf gummöse Herde in der Milz wohl mit Sicherheit gestellt werden kann. An eine Verwechslung mit 
Tuberculose ist bei der reichlichen schwielenbildung, dem Mangel von Erweichung and dem immerbin noch nicht hochgradigen käsigen Zerfall nicht zu denken, ebensowenig wie an verschrumpfte ältere Infarcte, da die Lage der Knoten und das Verhalten der Gefässe solchen gewiss nicht entspricht.

Wien, den 12. Juli 1880.

\section{Erklärung der von Herrn Dr. J. Heitzmann ausgeführten Abbildungen.}

Fig. 1. Mediane sagittale Durchschnittsfläche der Milz, a die Gummata, $z$ die käsigen Herde - natürliche Grösse.

Fig. 2. Durchschnitt durch die Grenzone zwischen den Gummen und dem angrenzenden Milzparenchym. a die käsigen Herde, $b$ das Milzparenchym. (Vergrösserung 10.)

Fig. 3. Gummagewebe. $a$ mehr centrale, $b$ mehr periphere Schichte (Hartnack Obj. VIII, Ocular 1.)

Fig. 4. Uebergang der Zellenbalken der Milz in das Gummagewebe. (Hartnack Obj. VII, Ocular 3.) 
